\title{
REPRESENTACIONES DE LIRAS EN LAS ESTELAS DECORADAS DEL BRONCE FINAL DE LA PENÍNSULA IBÉRICA
}

\author{
ALFREdo Mederos MARTín ${ }^{1}$ \\ Becario Postdoctoral de la Dirección General de Investigación Científica y Ténica \\ Ministerio de Educación y Ciencia \\ Department of Archaeology \\ University oBon soirf Bristol
}

\section{Resumen}

Las representaciones de liras de nueve cuerdas en las estelas decoradas del Bronce Final II-III de la Península lbérica presentan una cronología mucho más antigua de la que tradicionalmente se les ha atribuido mediante la búsqueda de paralelos fenicios o en el geométrico griego. Creemos que deben encuadrarse mejor en el Bronce Final II, $c a$. 1300-1150 A.C., correlacionándose con ejemplares de liras de siete cuerdas del Minoico Final IIIA o IIIB o del Heládico Final IIIC., ca. 1400-1190 A.C.

\begin{abstract}
The carvings of nine strings lyres in the Iberian Peninsula Late Bronze Age II-III have an older chronology than usually are know, looking for Phoenician or Geometric Greek parallels. We believe they must have better position in the Late Bronze Age II, ca. 1300-1150 BC, and correlations with seven strings lyres of the Late Minoan IIIAIIIB, ca. 1400-1190 BC o Late Helladic IIIC, ca. 1225/1185-1100 B.C.
\end{abstract}

La presencia de liras, o al menos de una lira en una estela decorada del Bronce Final de la Península Ibérica procedente de Tiña del Royo (Luna, Zaragoza) publicada por G. FaTás (1975), fue advertida por primera vez por M. BENDALA (1979, 36-37).

Según este autor, el referente más claro de la misma "sin tener en cuenta el número de cuerdas o la decoración", sería una phorminx del Geométrico Final de la segunda mitad del

\footnotetext{
${ }^{1}$ Queremos agradecer los comentarios al texto de R.J. Harrison
} 
S. VIII a.C. Esta lira, cuyo dibujo en una pyxis de estilo Laconio fue publicada por C. TsounTAS (1892, lám. 4/2), y es recogida también, a partir de una posterior publicación de S. WIDE (1899, 84, figura 42), en la síntesis de J.N. Coldstream (1968, XXIV, 217-218, lám. 26n) quien la interpreta dentro de una escena de danza masculina.

Sin embargo, a nuestro juicio, este referente de la lira presenta argumentos débiles. La escena, donde aparecen realmente dos liras, una de ellas casi totalmente perdida por la fractura en el vaso, pero que se observa mejor en el dibujo de Tsountas (1892, lám. 4/2), es conectada por Bendala exclusivamente en función de su "estructura", la forma semicircular de la caja de resonancia y la abertura de los brazos, pero la presencia de sólo 4 cuerdas frente a las 9 presentes en la estela de Luna las distancia definitivamente.

El paralelo expuesto por J.Mª. BLÁzQuEz (1983, 216, figura 3) de una terracotta fenicia del S. VIII a.C. procedente de Ashdod, es difícilmente admisible dada la tosquedad de la misma, aunque también parece advertirse que se trata de individualizar la presencia de unas 4 cuerdas.

Otro ejemplar que ha sido presentado como tal, procede de la estela de Los Llanos (Zarza Capilla, Badajoz) (ENríguez, 1982, 66-67, figura 2), pero dado su esquematismo es preferible no realizar demasiadas consideraciones sobre la misma. No obstante, según BLÁzQuEz (1983, 217, figura 4), el paralelo más próximo sería la lira que porta una estatuilla de bronce procedente de Monte Sirai (Cerdeña), que fecha a comienzos del S. VI a.C., si bien desde nuestro punto de vista quizás no sea del todo óptimo ya que, como el ejemplo de Zarza Capilla, se trata de una representación bastante esquemática de lira, en este caso por el soporte de bronce en que está fabricada.

En general, la búsqueda de paralelos por ambos autores ha estado mediatizado por las tesis que defienden. En el caso de Bendala $(1977,200)$ sobre la arribada a las costas meridionales andaluzas de emigrantes griegos del periodo geométrico que habrían aportado junto a nuevas armas, costumbres religiosas y funerarias, incluyendo el uso de la escritura y de instrumentos musicales, caso de las liras.

Por parte de BlázQuez (1985-86, 486), apoyando su hipótesis de vincular los registros gráficos de las estelas decoradas del Suroeste de la Península Ibérica, de cascos, escudos, fíbulas, espejos, carros o liras con el impacto del comercio fenicio en las poblaciones indígenas del interior. Estos modelos de liras "fenicias" serían utilizadas en las ceremonias fúnebres de ritual fenicio introducidas en la Península Ibérica (BLÁZquez, 1983, 218).

Resulta interesante observar cómo el propio BENDALA $(1979,37)$ correctamente admite que la presencia de 9 cuerdas implica tradiciones más antiguas del ámbito cretomicénico, diferentes de la lira homérica que ya cuenta con 4 cuerdas, idea que también recoge al sintetizar su argumento el trabajo de BLÁzQuez $(1983,213)$, haciendo ambos referencia al sarcófago de Hagia Triada, en el cual la lira representada cuenta con 7 cuerdas. Sin embargo, ninguno se plantea la necesidad de valorar con más detalle paralelos más antiguos o de otras áreas geográficas de los que las hipótesis concretas de ambos exigen.

Procedentes del ámbito egeo existe un tipo de lira con caja semicircular y brazos variables, bien rectos como en Nauplión, bien mayoritariamente formando un motivo de posibles cabezas de pájaros o serpientes enroscadas alrededor del mismo (tabla 1, figura 1). 


\begin{tabular}{|c|c|c|c|c|c|c|}
\hline Yacimiento & Provincia & Región & Pais & Soporte & Cronología & Bibliografia \\
\hline Luna & Zaragoza & Aragón & España & estela & Bronce Final II-III & $\begin{array}{l}\text { Fatas, 1975. 165-169. lám. 1-3: } \\
\text { Bendala, M., 1979. 36-37 }\end{array}$ \\
\hline Zarza Capilla & Badajoz & Extremadura & España & estela & Bronce Final II-III & Enriquez. 1982, 66-67, fig. 2 \\
\hline $\begin{array}{l}\text { Kalamión } \\
\text { Apokoronou, sep. } 1\end{array}$ & Chania & Creta & Grecia & cerámica. pyxis & Minoico Final IIIB & Tzedakis, 1970,111 , fig. 1 \\
\hline Hagia Triada & Heraklion & Creta & Grecia & sarcófago & Minoico Final IIIA & $\begin{array}{l}\text { Paribeni. 1903. 342-348; } \\
1904.713-719 \text { y } 1908,7-10\end{array}$ \\
\hline Hagia Triada & Heraklion & Creta & Grecia & fresco & Minoico Final IIIA & Paribeni. 1908. 68, fig. 21 \\
\hline Pilos & Mesenia & Peloponeso & Grecia & fresco & Heládico Final IIIA2 & Lang. 1969, 79-80, lám. 27 y 126 \\
\hline $\begin{array}{l}\text { Lagoumia sep. } 4 . \\
\text { Nauplión }\end{array}$ & Argólida & Peloponeso & Grecia & cerámica, crítera & Heládico Final IIIC & $\begin{array}{l}\text { Dragona-Latsoudi. } 1977 . \\
88 \text {, lám. 20a-b }\end{array}$ \\
\hline
\end{tabular}

Tabla 1. Representaciones de liras del Bronce Final, Heládico Final y Minoico Final de la Península Ibérica, Grecia y Creta. 


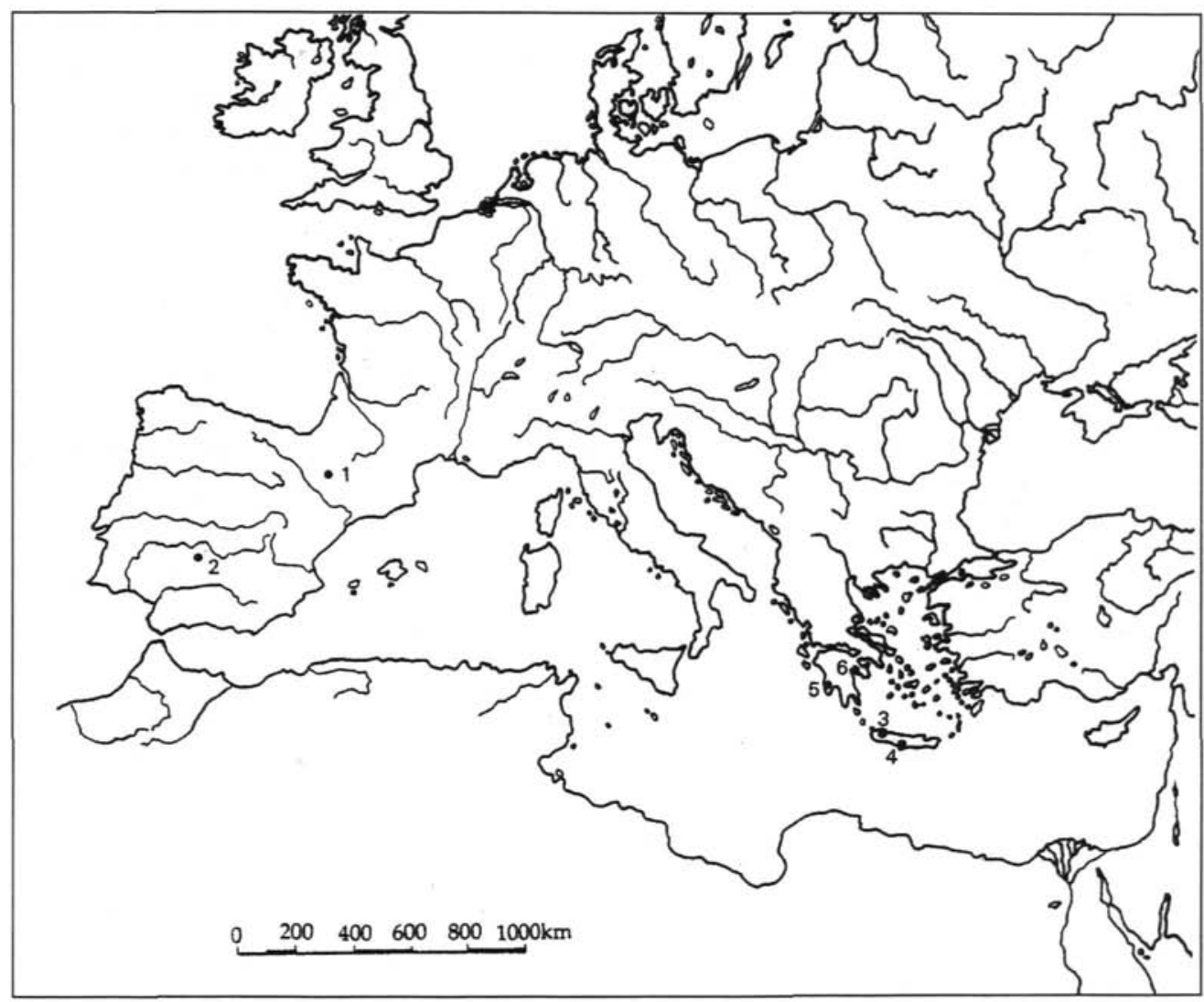

Figura 1. Distribución de las representaciones de liras en la Península Ibérica durante el Bronce Final II y IIIA, y en Grecia y Creta durante el Heládico y Minoico Final III. 1. Luna (Aragón), 2. Zarza Capilla (Extremadura), 3. Kalamión (Creta), 4. Hagia Triada (Creta), 5. Pilos (Mesenia), 6. Nauplión (Argólida). 
En general, todas ellas se tratan de representaciones fragmentarias que dificultan la interpretación del motivo de la lira, lo que es particularmente evidente en los más conocidos ejemplos de Hagia Triada y Pilos, y aunque al ser restauradas las pinturas del sarcófago y los murales se ofreció también una reconstrucción de las liras, no dejan de resultar discutibles tal como resalta B. LAWERGREN (1993, 63-64, figura 9), y queda de manifiesto al presentar los croquis de las mismas sin las partes reconstruidas.

Este hecho permite que en ocasiones se planteen nuevas reconstrucciones de estas liras que no dejan de ser igualmente discutibles (Platón, 1966, 212, figura 2a-c; VorRerter, 1975, pl. 22a-c).

Los ejemplares mejor conservados son los dos procedentes de dibujos en cerámicas, por conservarse completos sus dibujos, y reflejan la presencia de un tipo de lira y de otra posible. Una con perfil de caja semicircular, que también se observa con claridad en el sarcófago de Hagia Triada, caso del pyxis de Kalamión (TzeDaKIs, 1970, figura 1), y quizás otra que parece tener una forma más rectilínea y caja rectangular representada en la crátera de Nauplión (Dragona-LaTSOUdi, 1977, Lámina 20b) y de la que carecemos de otros dibujos para su contrastación lo que invita a la prudencia, pero que LAWERGREN $(1993,65)$ no admite, considerándola muy simplificada, para así defender la presencia de un único tipo con caja semicircular.

Sin embargo, resulta llamativo que dicho autor otorgue menos valor a las representaciones de Kalamión y Nauplión, considerándolas representaciones "pobres”, lo que es relativamente cierto si nos atenemos a los dibujos coloristas y detallistas de Hagia Triada o Pilos, pero que en la práctica son relativamente ineficaces ya que todas ellas carecen de un completo trazado de su juego de cuerdas.

El principal elemento de divergencia entre las liras cretenses y la de la Península Ibérica es la decoración animalística que presentan en sus brazos los ejemplares cretenses. Estos han sido interpretados como cuellos de cisnes (LANG, 1969, 80; VorreITER, 1975, 93-94), que será después el pájaro sagrado de Zeus y Apolo, o como flamencos (LAWERGREN, 1993, 63) por la extremada curvatura de los cuellos, aunque tampoco faltan referencias a otros animales como las gacelas (Evans, 1928, 834). En general se admite deșde A.J. Evans $(1901,105)$ hasta la actualidad (CARTER, 1995, 307) que los pájaros en la religión minoica del Bronce Medio y Final representan epifanías de los dioses y su representación en las liras según J.B. Carter sería la visualización de una presencia divina. Si en ocasiones las cabezas de los animales resultan más imprecisas, a veces a modo de serpientes enrolladas, en otros ejemplos como en la lira de Pilos es obvio que se trata de cabezas de aves. Es posible que se trate de una temática de tradición egea pues entre algunas estatuillas cicládicas que presentan liras destaca una de procedencia desconocida, hoy en el Metropolitan Museum de Nueva York, que parece tener también un motivo de pájaro (Rıchter, 1953: 156, pl. 6) asignable al Cicládico Inicial o Calcolítico. No obstante, una representación pintada, cuidadosa con los detalles, procedente del Xeste 3 de Akrotiri en la isla de Tera, del Cicládico o Bronce Final IA (Marinatos, 1984, 114, fig. 80) carece de este motivo animalístico, lo que nos advierte que aunque sea un motivo recurrente puede haber excepciones.

Respecto a las cuerdas, las liras de Hagia Triada, Nauplión y Kalamión presentan 7 cuerdas, mientras el segundo ejemplar de Hagia Triada, peor conservado tanto puede tener 
7 como 8 cuerdas, elemento técnico que las distancia claramente tanto de modelos contemporáneos egipcios y del Próximo Oriente, como de liras posteriores del ámbito del Egeo.

Uno de los datos más llamativos, que únicamente se puede reconocer en la "kithadoros" de Kalamión (TzEDAKIs, 1970, figura 1), es que las 7 cuerdas convergen en un pequeño cuerpo central de forma semicircular. Este detalle no se puede apreciar en las otras liras minoicas o micénicas por problemas de conservación o porque, en el caso de Hagia Triada, un brazo tapa dicho sector. No obstante, la lira de Nauplión, sobre la cual ya comentamos que pudiera responder a un modelo diferente o ser más simplificado por problemas de espacio al estar encuadrada entre dos estrechas bandas de la decoración general de la crátera, carece del mismo.

Si nos remitimos a la estela de Luna, que es la única en la que los detalles están relativamente cuidados para realizar cierto tipo de valoraciones, BENDALA $(1977,189)$ apuntaba correctamente que aunque en teoría se representan 16 líneas que cubren toda la lira, las únicas que realmente corresponden a las cuerdas musicales son las 9 centrales que convergen también en un cuerpo central, y que corresponderían a tiras de un material compacto para dar mayor consistencia al instrumento. Sin entrar en este tipo de valoraciones, está relativamente claro que dichas líneas resultan ajenas a la estructura básica de dicha lira porque, tanto en los brazos como en la base, se aprecia que donde se superponen a la estructura de la lira quien grabó dicha representación puso buen cuidado en interrumpir en dichos puntos el trazado de la decoración con líneas paralelas inclinadas y dientes de lobo, que adornan la lira.

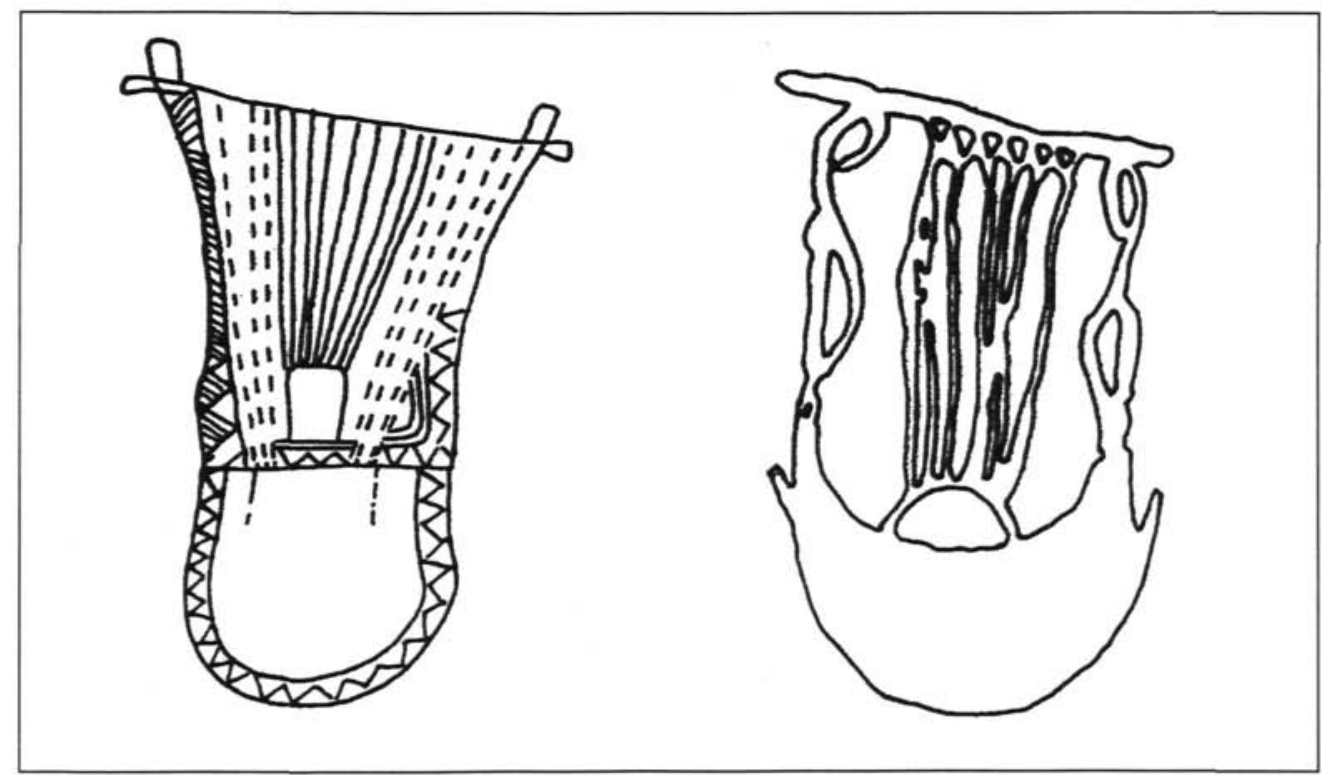

Figura 2. Liras de Luna (Zaragoza, España) y Kalamión (Creta, Grecia). A partir de Bendala (1977), con las líneas que no corresponden a las cuerdas de la lira en trazos discontínuos, y de la fotografia de Tzedakis (1970). 
Sin pretender presentar una simple relación mecánica entre la lira de Kalamión y la lira de Luna, ya que se diferencian claramente en el número de cuerdas, 7 en una y 9 en la segunda, no deja de resultar altamente llamativo los numerosos elementos de coincidencia entre ambas, tanto a nivel de la caja, forma semicircular y brazos ligeramente abiertos, como en la presencia de un cuerpo central donde convergen todas las cuerdas, que sólo conocemos en ambos ejemplares. Por otra parte, el gran número de cuerdas que presenta la lira de Luna podría igualmente ser indicativa de reproducir un modelo antiguo dentro de la evolución general de las liras.

En ambas regiones, además, están asociadas a individuos masculinos, si nos atenemos a tañedor de la lira en la procesión funeraria del sarcófago de Hagia Triada y el palacio de Pilos o a su asociación con un escudo con escotadura en V en la estela de Luna y a un individuo masculino portando una espada en Zarza Capilla, acompañado por escudo, lanza, carro y espejo; a su presencia en contextos relevantes, caso del Cuarto del Trono en Pilos, o en las propias estelas ibéricas; o a su inclusión como ajuar en algunas tumbas micénicas o minoicas, caso de Menidi, Micenas o Knossos, fabricadas en marfil o alabastro (Platon, 1966, 208-211, figura 1, lám. 67, 70-71). Y aunque el valor funerario de las estelas de la Península Ibérica resta aún por ser demostrado de manera definitiva, dada la perenne ausencia de contextos adecuados, no deja de ser un interesante punto de coincidencia.

Si observamos la cronología de los ejemplares cretenses y griegos, es evidente de que se trata de un modelo de amplia pervivencia temporal, al menos entre el Minoico Final IIIA y el Minoico Final IIIC, ca. 1435/1400-1100/1070 A.C.

\begin{tabular}{|lll|lc|}
\hline \multicolumn{2}{|l|}{ CRETA } & \multicolumn{2}{l|}{ PENÍNSULA IBÉRICA } \\
\hline M.F. IIIA 1 & $1435 / 1405-1390 / 1370$ & $1390-1370 / 1360$ & \multicolumn{1}{l|}{ B.F. IC } & $1425-$ \\
M.F. IIIA 2 & $1390 / 1370-1360 / 1325$ & $1370 / 1360-1340 / 1330$ & & $-1325 / 1300$ \\
M.F. IIIB & $1360 / 1325$ & $1340 / 1330-$ & B.F. IIA/ C.U. I & $1325 / 1300-1225$ \\
& $-1200 / 1190$ & -1190 & B.F. IIB & $1225-1150$ \\
M.F. IIIC & & $1190-1070$ & B.F. IIC/IIIA & $1150-1050$ \\
$\begin{array}{l}\text { Protogeométrico- } \\
\text { Subminoico }\end{array}$ & & & B.F. IIIA & $1050-950 / 925$ \\
\hline
\end{tabular}

Tabla 2. Dataciones según Manning, 1995:217, la primera columna y Warren y Hankey, 1989:169 la segunda, ambas para el Minoico Final cretense. Mederos (e.p.), para la Península Ibérica. Excepto Warren \& Hankey, dataciones absolutas calibradas según Stuiver y Reimer, 1993, cal. 3.0.3. 
En todo caso, si nos atenemos a la lira presente en la cerámica de Kalamion, asignable según TzEdaKIs $(1970,111)$ a inicios del Minoico Final IIIB, se corresponde bien cronológicamente con el Bronce Final IIA de la Península Ibérica, que es coetáneo con los primeros campos de urnas en el Noroeste ibérico, Cataluña y Aragón.

Algún autor como E. GaLÁn (1993, 21, 94, 103-104) sostiene que el valor de la estela de Luna debe ser relativizado por su "lejanía al centro de desarrollo del fenómeno" de las estelas decoradas del Suroeste de la Península Ibérica, sin embargo no duda en admitir la presencia de un "instrumento musical de cuerda" en dicha estela, e incluso acepta la presencia de otro "instrumento musical de cuerda" en la estela de Los Llanos (Zarza Capilla, Badajoz) que sí se encuentra en el centro geográfico de dicho fenómeno de las estelas, acompañado por espada, escudo con escotadura, lanza y carro.

Ya hemos planteado en otro trabajo (MEDEROS y HARRISON, e.p.) que al menos algunas de las estelas han de ser coetáneas al Bronce Final II dada la presencia de espadas pistiliformes en algunas de ellas, y aunque en ocasiones resulte difícil su interpretación, otros autores también las valoran en este mismo sentido (Almagro Basch, 1966, 152; Valiente y Prado, 1977-78, 377-378; COFFYN, 1985, 211; BARCELÓ, 1989, 192; MARTín MOMPEAN, 1992, 80) y particularmente generaliza dicho fenómeno C. BurGess (1992, 40).

Por otra parte, MARTÍN MOMPEAN $(1992,75,80)$ ha revalorizado correctamente la representación de una espada con cuatro remaches de la estela de Fóios inequívocamente de tipo Rosnoën, sin embargo al igual que inicialmente apuntó BARCELó $(1989,192)$ la interpreta como un rasgo arcaizante. No obstante, con las debidas precauciones que deben tomarse dado que se trata de momento de una única representación, creemos que no debe descartarse su atribución a un momento avanzado del Bronce Final IC, dada la llegada algo tardía de estas espadas a la Península Ibérica, en torno al 1350-1325/1300 A.C., lo que retrotraería asimismo la cronología de las estelas decoradas.

Apoyándonos en la exposición precedente, el primer argumento que queremos enfatizar es que probablemente hacia mediados del S. XIII A.C., coexisten durante el Bronce Final IIA de la Península Ibérica y el Minoico Final IIIB, liras con un elevado número de cuerdas, 9 en la Península Ibérica y 7 en Creta, que desde un punto de vista formal presentan notables puntos de contacto.

En segundo lugar, el mayor número de cuerdas implica un mayor número de notas musicales, lo que indica composiciones de relativa complejidad que quizás irían acompañadas por cánticos durante los rituales funerarios. La presencia de liras indica presumiblemente la posible existencia de un músico especialista, al menos durante parte del año o a tiempo parcial, ya que no hay constancia durante el Calcolítico o Bronce en el Mediterráneo Oriental o Próximo Oriente de miembros de la élite que toquen habitualmente instrumentos musicales, y sí de músicos con sus correspondientes instrumentos. En el caso de la estela de Luna, resulta difícil presuponer que se erigió a un individuo que simultáneamente era guerrero y músico, dada la presencia de un escudo con escotadura en $\mathrm{V}$.

Finalmente, apuntar que la presencia de nueve cuerdas implica un modelo musical evolucionado que está presente en las arpas de nueve cuerdas asirias, al menos desde la primera mitad del S. IX a.C., y posteriormente durante el periodo Babilónico (LAWERGREN Y 
GuRNEY, 1987, 51), se produce un incremento en el número de cuerdas hacia los que evolucionarán tipos derivados de la phorminx homérica geométrica, recuperando la tradición de las liras micénicas y minoicas.

\section{BIBLIOGRAFÍA}

Almagro Basch, M. (1966): Las estelas decoradas del Suroeste peninsular; Biblioteca Praehistórica Hispana VIII, CSIC, Madrid.

Barceló, J.A. (1989): "Las estelas decoradas del Sudoeste de la Península Ibérica", Tartessos. Arqueología Protohistórica del Bajo Guadalquivir, Ed. Aubet, Mª.E., Ausa, Sabadell, 189-208.

Bendala Galán, M. (1977): “Notas sobre las estelas decoradas del Suroeste y los orígenes de Tartessos", Habis 8, 177-205.

BEndala GaLÁN, M. (1979): "Las más antiguas navegaciones griegas a España y el origen de Tartessos", Archivo Español de Arqueología 52, 33-38.

Blázquez Martínez, J.Mª. (1983): "Las liras de las estelas hispanas de finales de la Edad del Bronce", Archivo Español de Arqueología 56, 213-228.

Blázquez Martínez, J.Ma. (1985-86): "Los escudos con escotadura en V y la presencia fenicia en la costa atlántica y en el interior de la Península Ibérica", Veleia 2-3, 469-497.

Burgess, C. (1991): "The East and the West: Mediterranean influence in the Atlantic World in the Later Bronze Age, c. 1500-700 B.C.", L'Age du Bronze Atlantique, Eds. Chevillot, C. y Coffyn, A., Association des Musées du Sarladais, Beynac-et-Cazenac, 25-45.

CARTER, J.B. (1995): “Ancestor Cult and the Occasion of Homeric Performance", The Ages of Homer: A Tribute to Emily Townsend Vermeule, Eds. Carter, J.B. y Morris, S.P., University of Texas Press, Austin, 285-312.

Coffyn, A. (1985): Le Bronze Final Atlantique dans la Péninsule Ibérique, Picard, Paris.

Coldstream, J.N. (1968): Greek Geometric Pottery. A survey of ten local styles and their chronology, Methuen, London.

Dragona-Latsoudi, A. (1977): "Mykinaïkos kitharodos apo ti Nauplia", Archaiologiki Ephemeis 116, 86-98.

Enríquez NavascuÉs, J.J. (1982): "Dos nuevas estelas de guerreros en el Museo Arqueológico Provincial de Badajoz", Museos 1, 65-68.

Evans, A.J. (1901): "Mycenaean Tree and Pillar Cult and Its Mediterranean Relations", Journal of Hellenic Studies 21, 99-204.

Evans, A.J. (1928): The Palace of Minos at Knossos II, MacMillan, London.

FATÁs, G. (1975): "Una estela de guerrero con escudo escotado en "V" aparecida en Cinco Villas de Aragón", Pyrenae 11, 165-169.

GalÁn Domingo, E. (1993): Estelas, paisaje y territorio en el Bronce Final del Suroeste de la Peninsula Ibérica, Complutum, Extra 3, Universidad COMPLUTENSE DE MADRID, MADRID.

LANG, M.L. (1969): The Palace of Nestor at Pylos in western Messenia. II. The frescoes, Princenton University Press, Princenton. 
LaWErgren, B. (1993): "Lyres in the West (Italy, Greece) and East (Egypt, the Near East) ca. 2000 to 400 B.C.", Opuscula Romana 19, 55-76.

LaWERgren, B. y GuRney, O.R. (1987): "Sound Holes and Geometrical Figures. Clues to the Terminology of Ancient Mesopotamian Harps", Irak 49, 37-52.

Manning, S.W. (1995): The Absolute Chronology of the Aegean Early Bronze Age. Archaeology, Radiocarbon and History, Monographs in Mediterranean Archaeology 1, Sheffield Academic Press, Sheffield.

Marinatos, N. (1984): Art and Religion in Thera. Reconstructing a Bronze Age Society, Athens.

Martín Mompean, J.L. (1992): "Nuevas aportaciones al estudio de dos estelas decoradas halladas en la cuenca sur del Duero (Beira Alta, Portugal)", Cuadernos de Prehistoria y Arqueología de la Universidad Autónoma de Madrid, 19, 67-93.

Mederos Martín, A. (e.p.): "La cronología del Bronce Final en Andalucía y su inserción en las secuencias atlántica y mediterránea”. Pyrenae.

Mederos, A. y Harrison, R.J. (e.p.): Patronazgo y clientela. Honor, guerra y festines en las relaciones sociales de dependencia del Bronce Final atlántico en la Península Ibérica, Pyrenae.

PARIBENI, R. (1903): "Lavori eseguita della Missione Archeologica Italiana nel palazzo e nella necropoli di Haghia Triada da 23 Febraio al 15 Luglio 1903", Rendiconti della $R$. Accademia dei Lincei 12, 5 S., 317-351.

PARIBENI, R. (1904): "Ricerchi nel sepolcreto di Haghia Triada presso Phaestos", Monumenti Antichi 14, 677-755.

PARIBENI, R. (1908): "Il sarcofago dipinto di Haghia Triada", Monumenti Antichi 19, 5-86.

Platon, N. (1966): "Minoike Lyra", Charisterion eis Anastasion K. Orlandon, Bibliotheke tes en Athenais Archaiologikes Hetaireias 54, vol. III, 208-226.

RichTER, G.M. (1953): Handbook of the Greek Collection, Metropolitan Museum of Art, New York.

Stuiver, M. y Reimer, P.J. (1993): "Extended 14C data base and revised calib 3.0 14C age calibration program", Radiocarbon 31, 215-230.

Tsountas, C. (1892): "Ek toi amikleoi”, Archaiologiki Ephemeis 31, 1-26.

TzEDAKIS, J. (1970): "Minoikoss kitharodos", Athens Annals of Archaeology 3, 111-112.

VAliente, J. y Prado, S. (1977-78): "Estelas decoradas de Aldea del Rey (Ciudad Real)", Archivo Español de Arqueología, 50-51, 375-388.

VORReITER, L. (1975): "The Swan-neck Lyres of Minoan-Mycenean Culture", The Galpin Society Journal 28, 93-97.

Warren, P. y Hankey, V. (1989): Aegean Bronze Age Cbronology, Bristol Classical Press, Bristol.

WIDE, S. (1899): "Geometrische vasen aus griechenland", Jabrbuch des Deutschen Archaologischen Instituts 14, 78-86. 